\title{
Synthesis of Polyaniline on an Aquatic Plant
}

\section{Aohan Wang, Ryosuke Kikuchi, Hiromasa Goto*}

\author{
Division of Materials Science, Faculty of Pure and Applied Sciences, \\ University of Tsukuba, Tsukuba, Ibaraki 305-8573, Japan \\ E-mail: gotoh@ims.tsukuba.ac.jp (H. Goto, Prof., Dr.)
}

Keywords: algae; diatom; ESR; SEM; Oedogonium sp.

\begin{abstract}
Polyaniline was synthesised on the surface of Oedogonium sp. in the water medium for producing a bio/synthetic polymer composite. Infrared absorption (IR) and electron spin resonance spectroscopy (ESR) measurements for the samples were carried out. Surface observation was carried out with the polarising optical microscopy (POM) and the scanning electron microscopy (SEM). Polymerisation reaction was progressed on the surface of Oedogonium sp. as a bio-template. Synthesis of PANI on a bacillariophyceae (diatom) was also carried out
\end{abstract}

\section{Introduction}

Algae bloom is a puzzled issue when discussing the environmental problems. Mass propagation of algae pollutes the water and sometimes causes negative impacts to normal ecosystem [1]. In the present circumstance, a solution that can exploit these nature products for industry is no less important than eradication. Various natural lives living in the rivers and the ponds possess complicated structure that still not been achieved artificially. These structures are suitable templates for production of composite materials. In the present study, we employ Oedogonium sp. as a bio-template in production of polyaniline. Polyaniline as a conducting polymer can be prepared in the water medium and shows good redox property, anti-corrosion, and optical activity [2,3]. The resultant composite of PANI/Oedogonium sp. possess a form of hollow fiber. The formation can improve processability of polyaniline.

\section{Experiment}

\section{Oedogonium sp.}

Oedogonium sp. was collected manually from a small stream in University of Tsukuba, Japan on March; 2015. Optical micrographs of Oedogonium sp. are shown in Figure 1. Oedogonium shows birefringence, as shown in Figure 1(a,b). Magnification image displays organelle of Oedogonium inside of cell Figure 1(c).

\section{Polymerisation on the Oedogonium sp.}

A solution of aniline and sulfuric acid in the water was stirred in the presence of Oedogonium sp. for 1 hour at room temperature. The mixture was cooled to $0{ }^{\circ} \mathrm{C}$ and stirred for $1 \mathrm{~h}$. Then, ammonium peroxodisulfate (APS) was slowly added into the mixture. After reaction for $24 \mathrm{~h}$, the resultant material was washed with a large volume of water, and methanol followed by filtration. The PANI/Oedogonium sp. was collected by filtration, and dried under vacuum (Table 1). Colour of the composite is dark emerald green. The biocomposites thus prepared are abbreviated as PANI/Oedogonium-n $(\mathrm{n}=1-4)$, as shown in Table 1. During the polymerisation process, these bio materials work as a template. The obtained composite appear like a planar sheet. 
Table 1. Polymerisation.

\begin{tabular}{ccccccc}
\hline Composite & $\begin{array}{c}\text { Aniline } \\
(\mathrm{g})\end{array}$ & $\begin{array}{c}\mathrm{APS}^{1} \\
(\mathrm{~g})\end{array}$ & $\mathrm{H}_{2} \mathrm{SO}_{4}(\mathrm{~g})$ & Oedogonium sp. $(\mathrm{g})$ & $\begin{array}{c}\mathrm{H}_{2} \mathrm{O}^{2} \\
(\mathrm{~mL})\end{array}$ & $\begin{array}{c}\text { Yield } \\
(\mathrm{g})\end{array}$ \\
\hline PANI/Oedogonium-1 & 0.2 & 0.2 & 0.2 & 1.14 & 200 & 0.23 \\
PANI/Oedogonium-2 & 0.5 & 0.5 & 0.5 & 1.09 & 200 & 0.36 \\
PANI/Oedogonium-3 & 0.1 & 0.1 & 0.1 & 1.07 & 200 & 0.20 \\
PANI/Oedogonium-4 & 1.0 & 1.0 & 1.0 & 1.00 & 500 & 0.53 \\
\hline
\end{tabular}

\footnotetext{
${ }^{1}$ Ammonium peroxodisulfate.
}

${ }^{2}$ Distilled water.

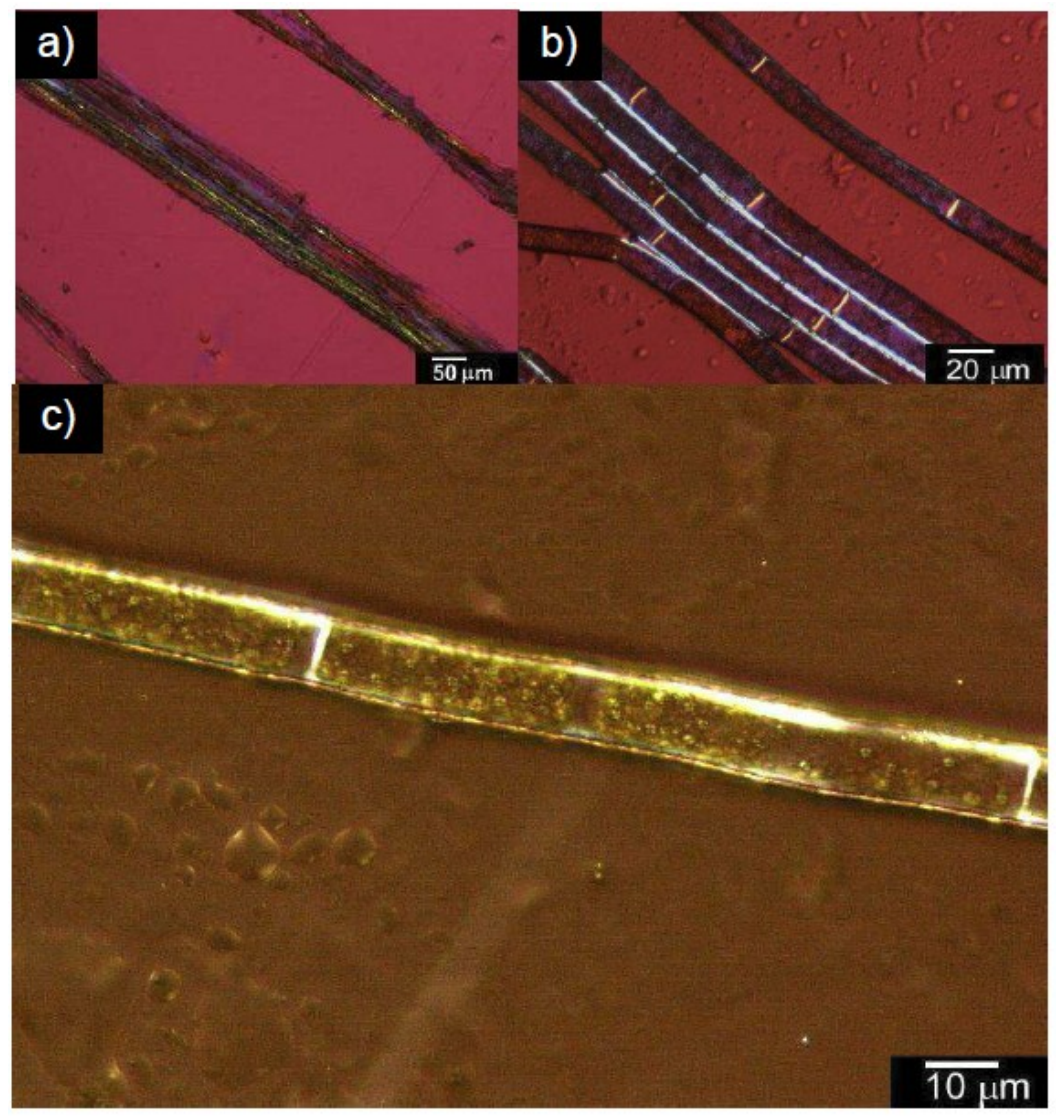

Figure 1. Optical micrographs of Oedogonium sp. (a,b): Polarising optical micrographs. (c): Magnification image (no cross nicol).

IR

IR spectra of PANI/Oedogonium sp. are shown in Figure 2. The PANI layer on the algae is emeraldine salt as a doped form. The monomer repeat unit of the PANI consists of benzenoid (B) and quinoid (Q) structures [4]. An absorption band due to quinoid structure is observable at around $1580 \mathrm{~cm}^{-1}$. Absorption bands ascribed to vibration of benzenoid structure is observed at $1490 \mathrm{~cm}^{-1}$. An intense absorption band at $3410 \mathrm{~cm}^{-1}$ is due to $\mathrm{OH}$ stretching. However, PANI/Oedogonium-4 shows no absorption of the $\mathrm{vOH}$ because infrared from the instrument could not reach inside of the sample, indicating the PANI layer of the PANI/Oedogonium 4 is thick. 


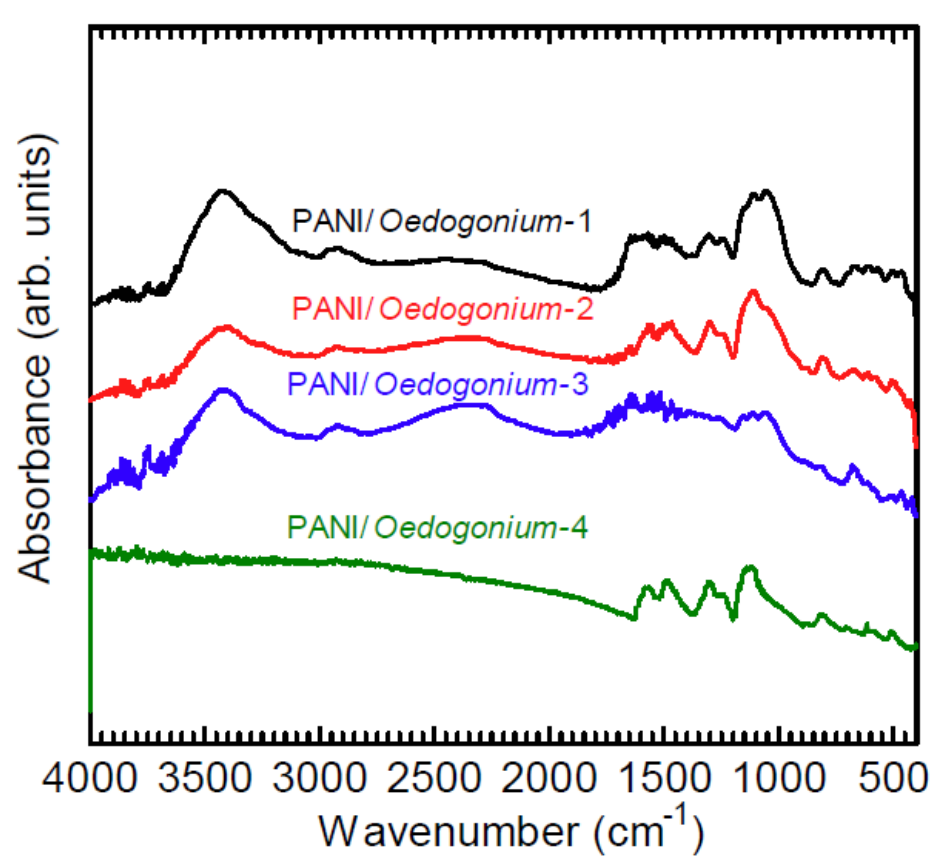

Figure 2. IR absorption spectra of PANI/Oedogonium sp.
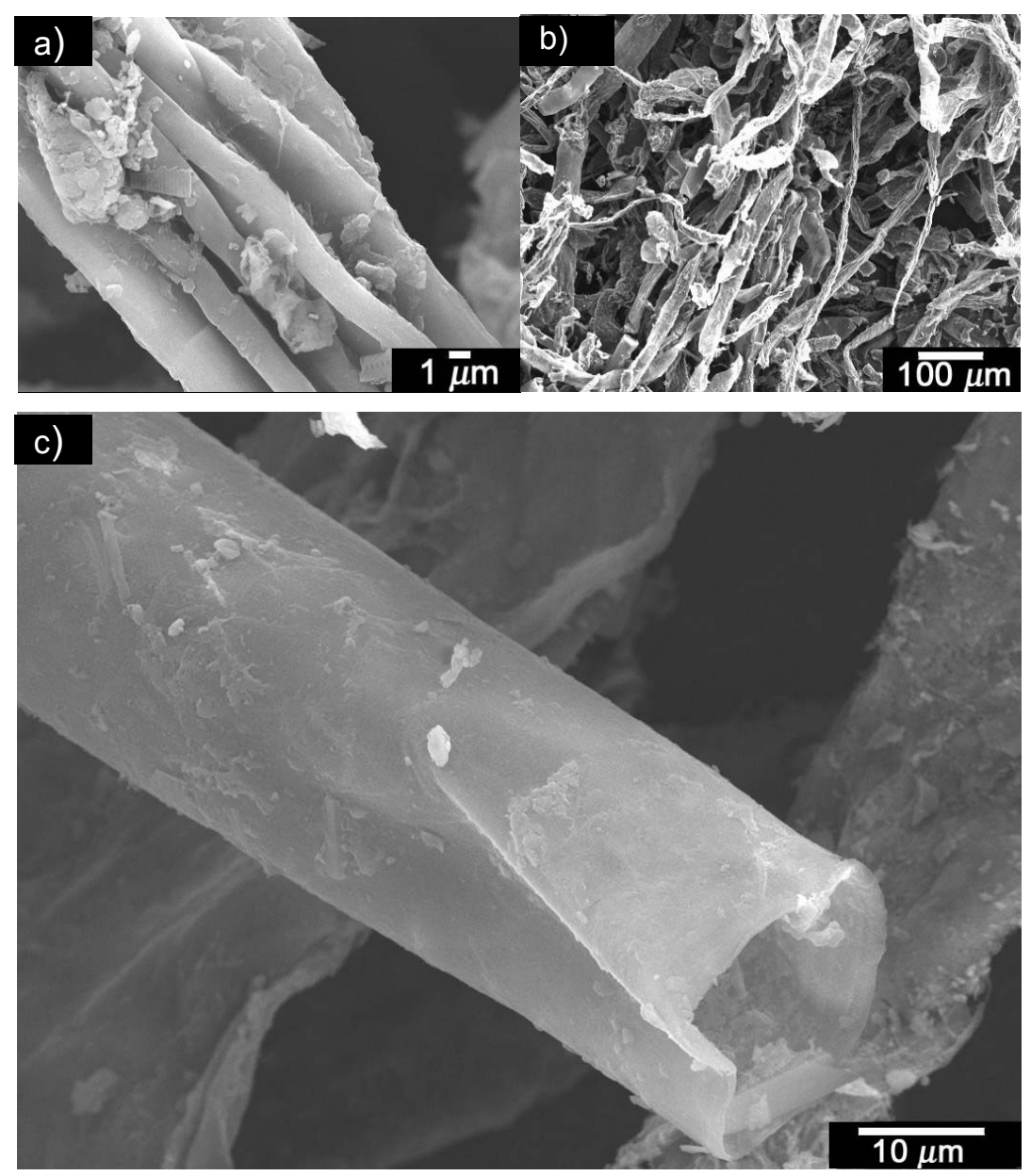

Figure 3. SEM images of PANI/Oedogonium-1. (a),(b): Low magnification images. (c): Magnification images. 
Scanning Electron Microscopy observation

Figs. 3,4 show scanning electron microscopy (SEM) images of PANI/Oedogonium-1 and PANI/Oedogonium-3, respectively. The samples in micro-scale show thread-like structure with polyaniline adhered on the surface. The form of the algae is retained after deposition of PANI on the surface. Vacuum drying not only removed the water contained in polyaniline powder but also evacuated the water inside of the algae, which gives the composite a hollow tube form, as shown in Figure 3(c). Although the composite forms cylindrical structure in the water, the dried samples are more like deflated tubes, which indicates that the resultant composite have hollow structure. These synthesised composite are unbranched, and some of them are sheet-like structure in macro-scale, where these unbranched composites stick to each other in different directions. Figure 5 shows a plausible structure of the bio-template polymerisation.

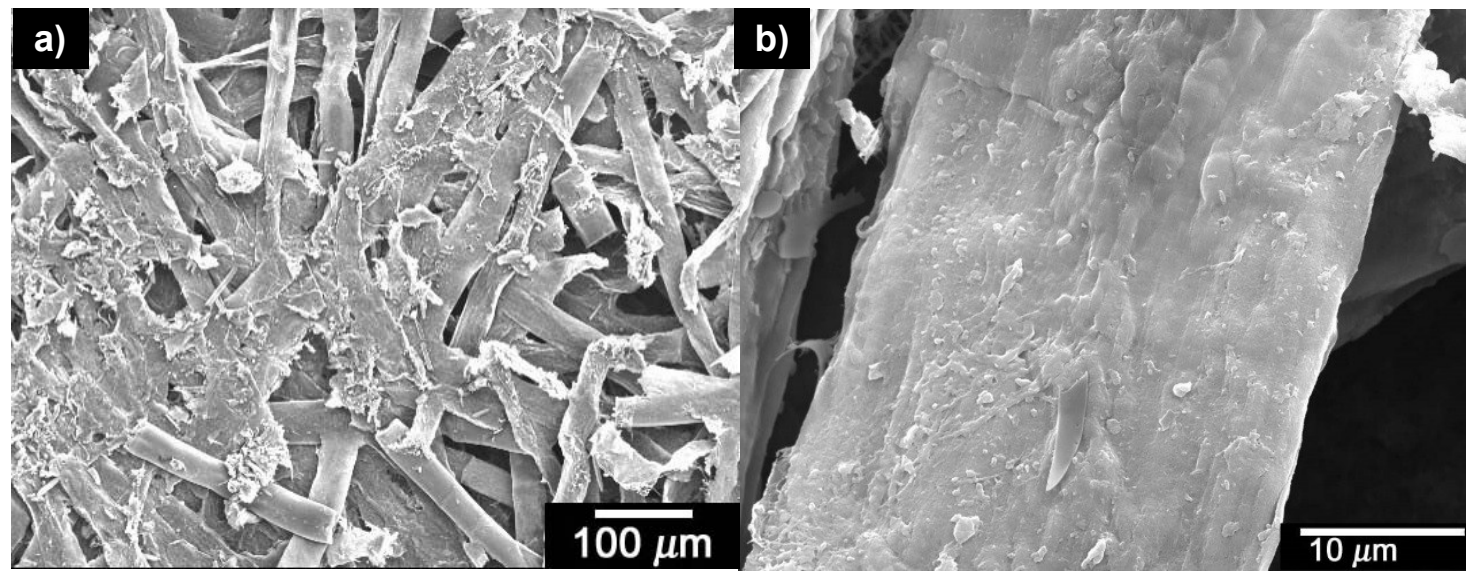

Figure 4. SEM images of PANI/Oedogonium-3. (a): Low magnification.

(b): High Magnification image.

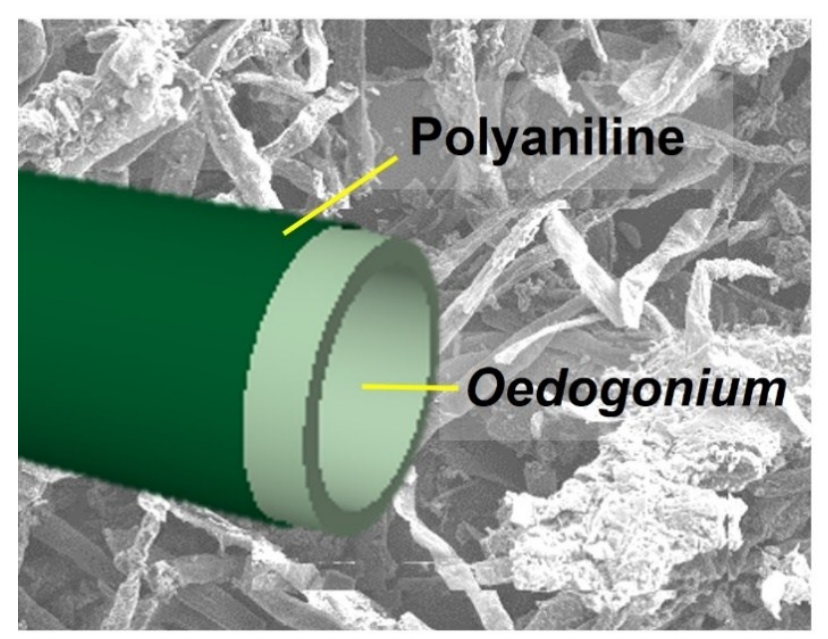

Figure 5. A plausible structure of the bio-template polymerisation resultant. PANI adheres on the surface of Oedogonium tube.

Bacillariophyceae (diatom, [5]) was also found under the SEM observation in the sample. These micro-sized organisms could not be removed completely, and as a result PANI/bacillariophyceae was partly observed. The SEM images of PANI/bacillariophyceae are shown in Figure 6. Combination of surface fine structure of the bacillariophyceae and PANI as a 
conducting polymer produces new ordered materials. This concept is belonged to neither bottom up technology nor top down technology.

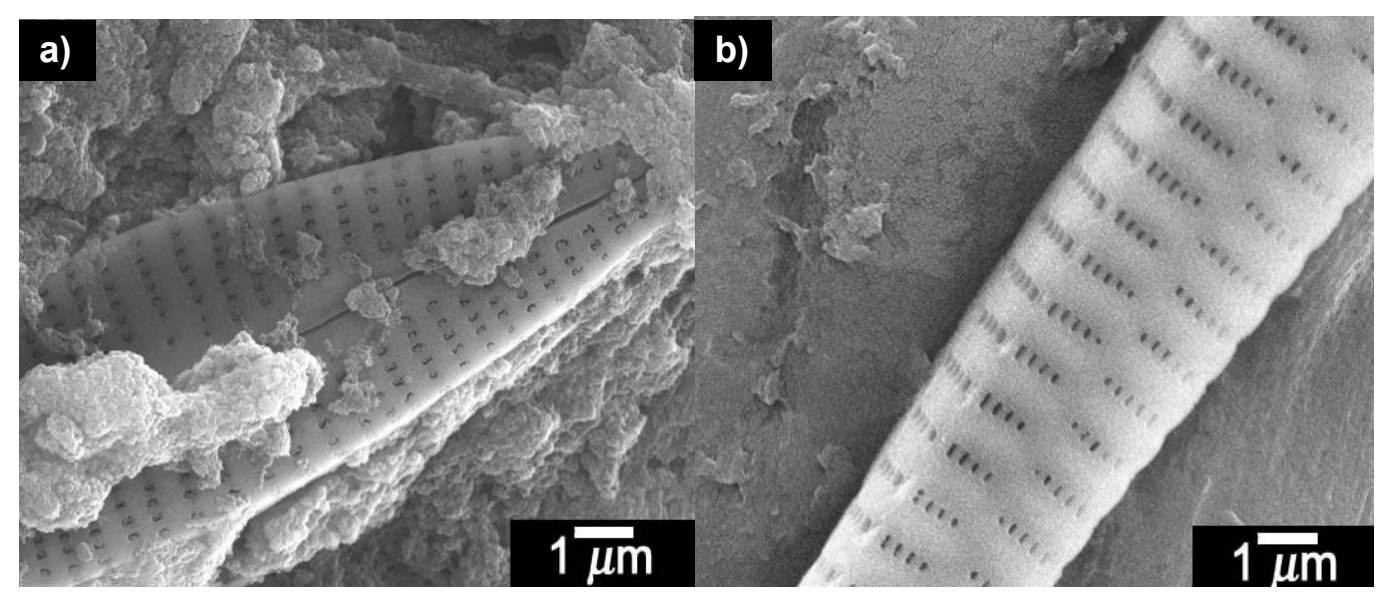

Figure 6. SEM images of PANI/bacillariophyceae (diatom) (a): Low magnification.

(b): Magnification image.

ESR

Electron spin resonance (ESR) spectrum of PANI/Oedogonium-4 is shown in Figure 7. The signal of the ESR demonstrates existence of radicals form polarons (radical cations) as a charge carrier of the doped form of PANI.

\section{Electrical conductivity}

Electrical conductivity of the PANI/Oedogonium-4 was evaluated with a four-probe method. Conductivity of PANI/Oedogonium-4 is to be $8.3 \times 10^{-3} \mathrm{~S} / \mathrm{cm}$, indicating the sample thus prepared can be referred to as electrical conductive bio-composites.

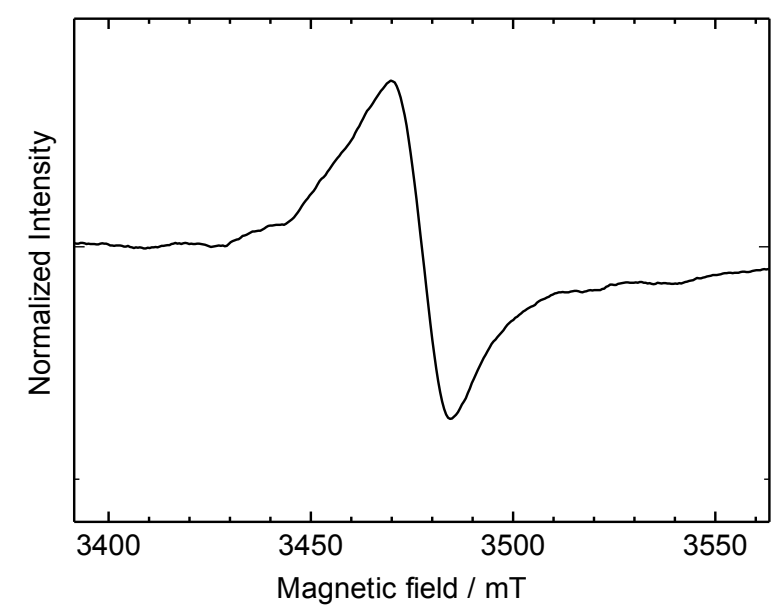

Figure 7. Electron spin resonance (ESR) spectroscopy measurement results.

\section{Conclusions}

We synthesized PANI/Oedogonium sp. and PANI/bacillariophyceae as bio-composites. Combination of micro-structure of algae and diatom allows to producing conducting polymers having fine structures. The bio/conductive polymer composites can be produced in a sheet form. They may be applied for practical use, such as flexible conductive sheet or antistatic sheet. 


\section{Techniques}

IR spectra of the samples were obtained with a JASCO IR300. SEM observations performed with a JEOL JSM-7000F. ESR measurements were recorded on a Bruker EMX-T ESR spectrometer. Electrical conductivity was measured by four-probe method using Mitsubishi Chemical Analytech LORESTA-GP MCP-T610.

\section{Author Contributions}

Goto collected Oedogonium and diatom, performed synthesis of the bio-composites, and observed the samples with the optical microscopy. Kikuchi performed ESR, IR, and electrical conductivity measurements. Wang observed surface structure of the samples with the SEM. She also carried out the IR measurements of the samples.

\section{Acknowledgments}

We would like to thanks Research Facility Centre for Science and Technology, University of Tsukuba, and Glass workshop of University of Tsukuba. SEM images were obtained in National Institute for Materials Science (NIMS) microstructural characterisation platform. We thank Dr. Takeguchi, Station Director, Transmission Electron Microscopy Station, NIMS.

\section{References}

[1] R.L.Fletcher, The occurrence of "green tides" - a review, 123 (1996) Ecological Studies, Marine Benthic Vegetation: Part A, 7-44.

[2] G.G. Wallace, P.R. Teasdale, G.M. Spinks, L.A.P. Kane-Maguire, Conductive electroactive polymers: Intelligent polymer systems, third edition. 2008, CRC Press, NY.

[3] D. Marsitzky, K. Mullen, 20 Years of "Synthetic Metals"- the role of synthesis, in: P. Bernier, S. Lefrant, G. Bidan (Eds), Advances in synthetic metals, twenty years of progress in science and technology, Elsevier Science SA, 1999, Switzerland, pp. 1-97.

[4] J. Tang, X. Jing, B. Wang, F. Wang, Infrared spectra of soluble polyaniline, Synth. Met., 24 (1988) 231-238.

[5] I. Inoue, The natural history of algae: Second edition: Perspective of three billion years evolution of algae, earth and environment, Tokai University Press, 2007, Kanagawa, Japan. 